\title{
Meal-stimulated Release of Methionine-Enkephalin into the Canine Jejunal Lumen
}

\author{
Samuel R. Money, Andy Petroianu, Alan R. Gintzler, and Bemard M. Jaffe \\ Departments of Surgery and Biochemistry, State University of New York Health Science Center at Brooklyn, Brooklyn, New York 11203
}

\begin{abstract}
Application of enkephalins to the luminal surface of the bowel augments intestinal absorption. However, to date, endogeneous enkephalins have not been demonstrated within intestinal luminal fluid. To determine whether enkephalins are present in the intestinal lumen, five adult dogs had $25-\mathrm{cm}$ chronic jejunal Thiry-Vella loops constructed. Dogs were studied in the awake, fasted state. Jejunal loops were perfused with isoosmotic, neutral Krebs buffer containing protease inhibitors. After basal sampling, the dogs received a high fat meat meal. Collections were made during the meal and for $60 \mathrm{~min}$ postprandially. Luminal met-enkephalin levels were determined by radioimmunoassay and confirmed by HPLC. HPLC separation of luminal samples demonstrated two immunoreactive peaks which co-eluted with pure met-enkephalin and met-enkephalin-sulfoxide. Basal met-enkephalin outputs averaged $52 \pm 13 \mathrm{ng} / \mathrm{min}$. The meal significantly increased mean luminal met-enkephalin output to $137 \pm 71 \mathrm{ng} / \mathrm{min}$. During the initial 20-min postprandial period, output remained elevated $(180 \pm 73$ $\mathrm{ng} / \mathrm{min}$ ), after which it returned to basal levels. We conclude that met-enkephalin is present in the jejunal lumen, and that luminal release of this opioid is augmented by a meal.
\end{abstract}

\section{Introduction}

The antidiarrheal properties of the opiates have been known for greater than 2,000 years (1). Until recently, the mechanism of this effect was thought to be solely due to induced inhibition of intestinal motility via their ability to inhibit the release of excitatory neurotransmitters such as acetylcholine (2), serotonin (3), and substance $P$ (4). However, in the past decade, it has become evident that opioids not only exert effects on motility, but also influence intestinal absorption (5-9).

In 1975, Hughes and colleagues (10) isolated and identified two pentapeptides from the brain which bound to opioid receptors. These peptides, recognized as endogenous opioids, were named enkephalins, i.e., methionine-enkephalin and leucine-enkephalin. One year later, these peptides were also identified in the gastrointestinal tract. Met-enkephalin immunoreactivity $(E L I)^{1}$ has been identified in both the myenteric

Address correspondence to Dr. Jaffe, Professor and Chairman, Dept. of Surgery, Box 40, SUNY-Health Science Center at Brooklyn, 450 Clarkson Ave., Brooklyn, NY 11203.

Received for publication 4 June 1987 and in revised form 11 August 1987.

1. Abbreviation used in this paper: ELI, enkephalin-like immunoreactivity.

J. Clin. Invest.

(C) The American Society for Clinical Investigation, Inc. $0021-9738 / 88 / 03 / 0822 / 04 \$ 2.00$

Volume 81, March 1988, 822-825 and submucosal plexus, as well as in cells of the intestinal mucosa $(11)$ of many mammalian species $(12,13)$ including man (14). Quantitative analysis of met-enkephalin in the gastrointestinal tract has demonstrated that the highest concentrations of the opioid are in the upper gut, and specifically in the stomach, duodenum, and upper jejunum. Receptors for these endogenous opioids have been demonstrated in the same regions (15-17).

In vitro studies have demonstrated that the application of opiates to the serosal side of the mucosa increases the absorption of water and electrolytes across the intestinal mucosa (1, 15-17). In an in vivo model, Fogel and Kaplan (8) demonstrated that perfusion of the intestinal lumen with a solution containing met-enkephalin analogues also increased fluid absorption. In studies with opiate antagonists, it was also demonstrated that endogenous opiates modulated intestinal handling of fluids and electrolytes. Extensive work with antagonists of specific receptor types indicated that these effects are mediated by the delta class of opiates, for which met-enkephalin is thought to be the endogenous ligand (9). The study presented here was designed to quantitate the presence of endogenous met-enkephalin in intestinal luminal fluid, and to characterize its release by a physiological stimulus, i.e., a meal.

\section{Methods}

Five adult female mongrel dogs (18-26 kg) had chronic $25-\mathrm{cm}$ ThiryVella loops constructed in the proximal jejunum as previously described (18). The dogs received perioperative streptomycin and penicillin for $48 \mathrm{~h}$ and were permitted a 2-wk recovery period postoperatively. All dogs remained healthy for the duration of the experiment and did not lose weight. Animals were studied in the awake state after an overnight fast, and were allowed free access to water.

The Thiry-Vella loops were perfused with Krebs buffer (concentrations in millimolars: $\mathrm{NaCl}, 118 ; \mathrm{KCl}, 4.7 ; \mathrm{CaCl}_{2}, 2.5 ; \mathrm{NaHCO}_{3}, 25$; glucose, $11, \mathrm{MgCl}_{2}, 1.2 ;$ and $\mathrm{NaH}_{2} \mathrm{PO}_{4}, 18.5$ ) in a peristaltic direction at $1 \mathrm{ml} / \mathrm{min}$. The perfusate contained $10 \mu \mathrm{M}$ captopril, $0.3 \mu \mathrm{M}$ thiorphan, $10 \mu \mathrm{M}$ bestatin, and $2 \mu \mathrm{M}$ L-leucyl L-leucine; all were added to protect against the action of proteases $(19,20)$. Neither the buffer itself nor the added chemicals interfere nonspecifically with the met-enkephalin RIA (19). The loops were perfused initially for a 60 -min equilibration period. After this, five 5-min effluent basal sample collections were obtained. The dogs were then fed a high protein, high fat meal (Alpo, $397 \mathrm{~g}$ ). Effluent collections were made during the meal and for the next 125 -min postprandial periods. All samples were collected in polyethelyene tubes on ice and centrifuged at $1,500 \mathrm{rpm}$ at $4^{\circ} \mathrm{C}$ for $15 \mathrm{~min}$ to remove mucus and particulate matter. The samples were then frozen and stored for $<14 \mathrm{~d}$ at $-80^{\circ} \mathrm{C}$.

Met-ELI was quantitated by RIA using the procedure originally described by Clark and Smith $(21,22)$. The rabbit anti-enkephalin antisera are $\mathrm{COOH}$-terminal specific; they recognize primarily met-enkephalin but its oxidized form, met-enkephalin sulfoxide, is equipotently bound. Leu-enkephalin and $\beta$-endorphin crossreact $<0.2 \%$. All assays were performed in duplicate in polypropylene tubes, with antisera used at a 1:500 final dilution. A standard curve $(50,100,250,500$,

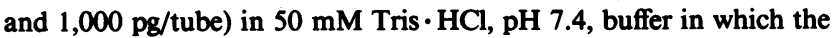
percentage of inhibition of binding of tritiated met-enkephalin (New 
England Nuclear, Boston, MA, $39.3 \mathrm{Ci} / \mathrm{mmol}$ ) was plotted against the $\log$ of unlabeled met-enkephalin in each reaction tube, was generated. The minimal detectable concentration of met-enkephalin was $25 \mathrm{pg}$, at which $8 \%$ inhibition of binding was observed. Bound- and free-labeled met-enkephalin were separated by filtration through mixed cellulose acetate, cellulose nitrate filters $(0.45 \mu \mathrm{m})$ under negative pressure. Radioactivity was quantitated by liquid scintillation spectroscopy.

The chemical nature of the ELI was confirmed by combining high pressure liquid chromatography (HPLC) fractionation and RIA detection $(19,20)$. HPLC fractionation was accomplished by injection of 20 $\mu l$ of either standard opioids (Peninsula Laboratories, Inc., Belmont, CA) or luminal effluents onto a $15-\mathrm{cm}$ reverse-phase $\mathrm{C}-18$ column (5 $\mu \mathrm{m}$, Nova-pak; Waters Assoc., Div. of Millipore Corp., Milford, MA). The column was eluted at $1 \mathrm{ml} / \mathrm{min}$ with a mobile phase containing $0.08 \%$ trifluroacetic acid throughout, and a linear gradient of acetonitrile ranging from 4 to $70 \%$. Ultraviolet (UV) absorption at $210 \mathrm{nM}$ was used to monitor elution of the standards. To verify the authenticity of the luminal met-enkephalin, 30-s collections were lyophilized to dryness and then subjected to RIA for quantification of ELI.

Statistical analyses were performed using the paired $t$ test applying the Bonferroni method. Due to interanimal variability, basal values were normalized and the mean percent increase characterized. In addition, integrated amounts of met-enkephalin released were quantitated by subtracting the basal from the postcibal (experimental) values and summing the increments for the $60 \mathrm{~min}$. Using these techniques, the significance of the increments representing luminal release was determined. Data are presented as mean \pm SEM and $P$ values of $<0.05$ were considered significant.

\section{Results}

Basal met-enkephalin output into luminal perfusates averaged $52 \pm 13 \mathrm{ng} / \mathrm{min}$ (mean $\pm \mathrm{SEM}$ ). During the meal, met-enkephalin outputs increased to $137 \pm 71 \mathrm{ng} / \mathrm{min}$. By normalizing the basal values, these data represent a mean increase of $159 \pm 36 \%$ over basal $(P<0.01)$. All five dogs demonstrated a prompt increase $(<5 \mathrm{~min})$ in luminal enkephalin release during the meal. Met-enkephalin outputs peaked at 10 min postprandially $(190 \pm 66 \mathrm{ng} / \mathrm{min})$ and thereafter slowly declined over the duration of the experiment (Fig. 1). The mean integrated

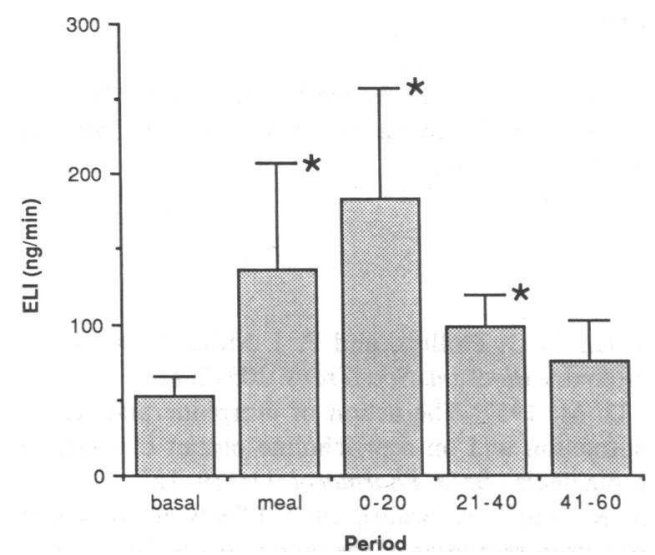

Figure 1. The effects of a meal on intraluminal met-enkephalin output. Luminal effluents were collected at 5-min intervals over a 25min period before exposing the animals to food, during the meal, and for $60 \mathrm{~min}$ after the meal. Each collection was assayed individually and the values pooled to establish the postprandial 20-min data points. The outputs during the meal and at $0-20$ and $21-40 \mathrm{~min}$ after the meal were significantly $(P<0.05)$ greater than those of the basal period. postprandial enkephalin output was $5.4 \pm 0.8 \mu \mathrm{g}(90 \pm 13 \mathrm{ng} /$ min), representing a $201 \pm 55 \%$ increase over the basal rate of release $(P<0.01)$.

Chemical identification of ELI was confirmed by combining high pressure liquid chromatographic fractionation with RIA detection. The HPLC conditions used permitted separation of met-enkephalin, met-enkephalin sulfoxide, and leu-enkephalin from each other and from other commonly occuring opioids such as $\alpha$-, $\beta$-, and $\gamma$-endorphin $(19,20)$. This procedure was used to fractionate luminal effluents from basal, meal-stimulated, and pooled postprandial periods before RIA quantitation. The separation of effluents from both the meal periods and the postprandial periods demonstrated significant increases compared with the basal period. In all instances, enkephalin luminal output eluted off the column in only two major peaks, one with the same retention time as met-enkephalin, the other with the same retention time as met-enkephalin sulfoxide (Fig. 2). The meal-stimulated release of ELI into luminal perfusates was still observed when the RIA was performed subsequent to fractionation by HPLC.

\section{Discussion}

In this study we have demonstrated the presence of met-enkephalin in the intestinal lumen. We have also identified a physiological stimulus which releases this peptide into the lumen, i.e., a meal. Prior studies have demonstrated the effects of topically applied met-enkephalin on the gut mucosa (5-7). Treatment of the mucosa with opioids increases the rate of fluid absorption compared with nontreated controls. This finding is in agreement with in vivo studies which show that met-enkephalin analogues increase absorption in intestinal loops (8) and that topically applied opiate antagonists reduce absorption $(6,8)$. These observations suggest a possible physiologic role for the endogenous opioids.

The canine jejunal Thiry-Vella loop was an appropriate model for these studies. Clarke and associates have demonstrated that exteriorized canine intestinal loops maintained both normal histology and functional activity (23). While screening electron microscopy in other species, such as rat, has demonstrated subtle alterations in surface morphology, these changes occur progressively with time and are more intense in the distal intestine (24). Our studies used proximal jejunum and were completed within three weeks. In addition, we have previously used these loops to document physiologic luminal release of serotonin and substance $P$ and to demonstrate specific neural control mechanisms for control of this release (25).

Note that the augmented release of met-enkephalin into the intestinal luminal perfusates occurred during the meal itself and preceded the entry of food into the intestine. This suggests that any change in gut function that is induced occurs in anticipation of enkephalin release into the intestinal lumen; changes must be secondary to neural or humoral stimuli rather than a direct action of food on the small intestine. In addition, this observation documents that the luminal met-enkephalin is of endogenous intestinal origin. Both the rapidity of the release (within minutes) and the total instability of the opioid in plasma suggest that the met-enkephalin could not have been absorbed and transported via the bloodstream to the isolated loop.

The locus of met-enkephalin's action on absorption remains obscure. In isolated mucosal studies, treatment of the 


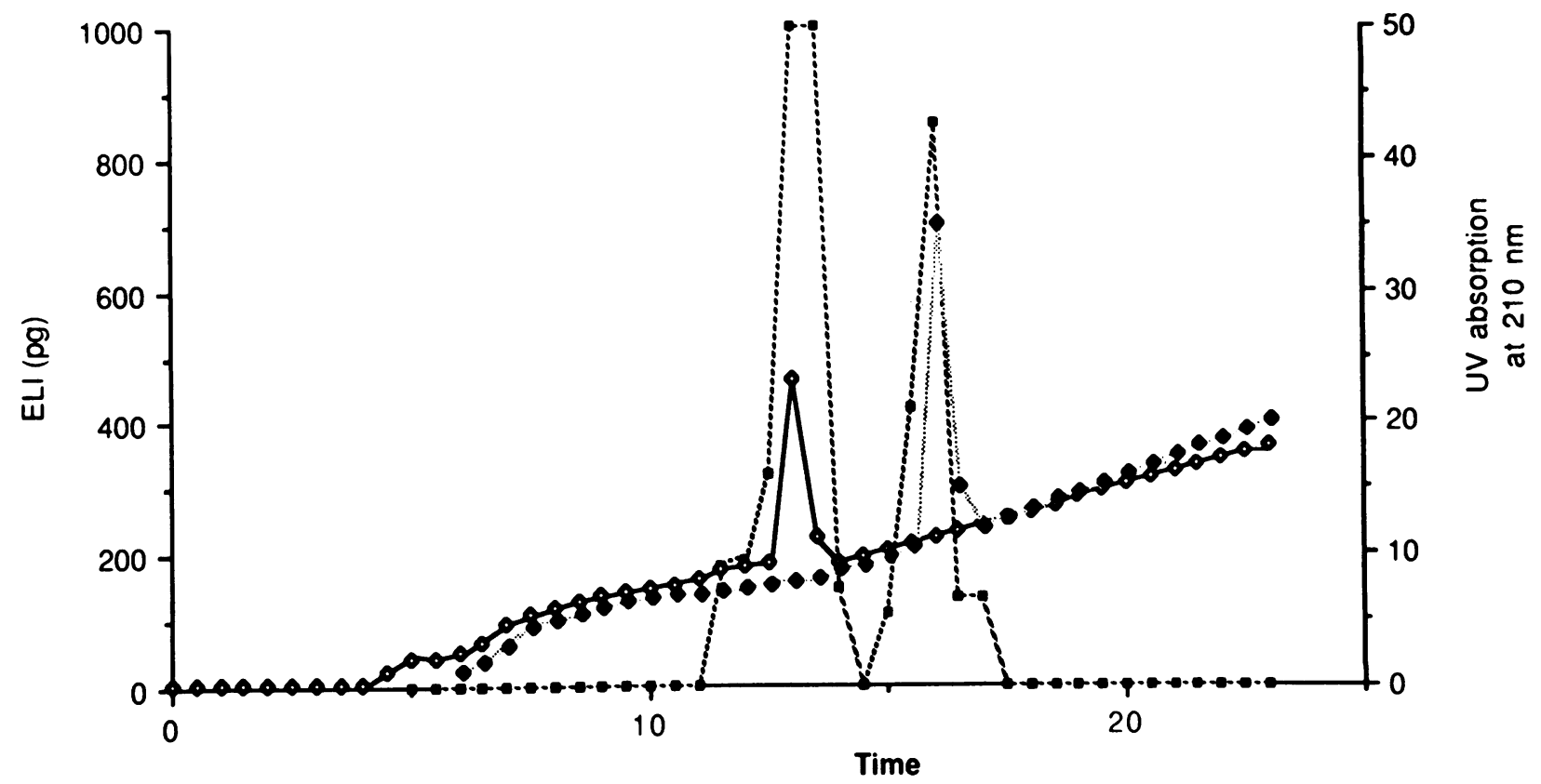

Figure 2. High pressure liquid chromatographic fractionation of metELI in jejunal luminal effluents. For comparison, the elution profiles (retention times) of met-enkephalin and its sulfoxide form are indicated by the absorption spectra at $210 \mathrm{~nm}(\diamond$, met-enkephalin sulfox-

submucosal side of the tissue with opiates demonstrated a significant effect on the potential difference and short circuit current across the tissue $(5,6)$. In contrast, application to the mucosal surface of the tissue demonstrated no change in either characteristic. This suggests that the ability of opiates to influence potential difference and short circuit currents is mediated via actions on the submucosal plexus or on the basal portion of the mucosal cells. In support of the submucosal plexus being the primary site of action, the enkephalin effect can be blocked by addition of tetrodotoxin, suggesting a preganglionic effect of met-enkephalin (6). In support of a possible direct enterocyte effect, receptor binding studies have demonstrated direct binding of leu-enkephalin to isolated mucosal cells. Moreover, enkephalins have been shown to stimulate intracellular cAMP levels (26) in intact isolated mucosal cells, suggesting a cellular mechanism. A paracrine effect must also be considered. Enterochromaffin cells contain receptors on their luminal surface. By activating these receptors, a second messenger may be released which modifies absorption. Thus, multiple mechanisms exist whereby luminal enkephalins can influence intestinal function.

The routes of delivery of the opioids alter the observed effects of these compounds. When the drugs are in direct contact with the gastrointestinal mucosa, significant changes in the rate of absorption have been noted (5-9). However, when similar drugs are given systemically, only motility was shown to be affected $(27,28)$ and there was no effect on the rate of absorption of water and electrolytes. This suggests that topical intestinal application may modify absorption while systemic opiates modulate only intestinal motility.

The precise role(s) of luminal enkephalin cannot be deduced from the present experiments; however, possible roles do exist for this luminal pentapeptide. In fact, met-enkephalin may modulate a variety of physiological functions in the sub-

ide; $\diamond$, met-enkephalin). The effluents obtained during the meal period were fractionated by HPLC as described in Methods. Each timed collection was lyophilized and the entire content of ELI was quantitated by radioimmunoassay ( $\boldsymbol{\square})$.

mucosal and/or the myenteric plexus of the small intestine. It may also act on the basal side of the enterocyte as a local paracrine modulator, and the opioid peptide may be cleared into the intestinal lumen after its physiological action. The sources of luminal met-enkephalin are unknown. However, whether it is directly secreted into the lumen, is cleared into the lumen after inducing on action elsewhere, or even if it "overflows" into the lumen from the blood, its presence in the intestinal lumen suggests additional regulatory actions for this peptide.

\section{Acknowledgments}

This research was supported in part by the Foundation for Surgical Education and Investigation, Brooklyn, NY, and by a grant from the National Institute on Drug Abuse.

\section{References}

1. Sandhu, B. K., A. D. Phillips, and P. J. Milla. 1984. Opiate agonists and enterotoxin. Biochem. Soc. Trans. 205-208.

2. Paton, W. D. M. 1957. The action of morphine and related substances on contraction and on acetylcholine output of coaxially stimulated guinea pig ileum. Br. J. Pharmacol. 11:119-127.

3. Gintzler, A. R., and J. A. Scalisi. 1982. Effects of opioids on noncholinergic excitatory responses of the guinea pig isolated ileum: inhibition of release of enteric substance P. Br. J. Pharmacol. 75:199205.

4. Gintzler, A. R. 1979. Serotonin participation in gut withdrawal from opiates. J. Pharmacol. Exp. Ther. 211:7-12.

5. Mc Kay, J. S., B. D. Linaker, and L. A. Turnberg. 1981. Influence of opiates on ion transport across rabbit ileal mucosa. Gastroenterology. 80:279-284.

6. Dobbins, J., L. Racusen, and H. J. Binder. 1980. Effect of D-ala- 
nine methionine enkephalin amide on ion transport in rabbit ileum. $J$. Clin. Invest. 66:19-28.

7. Vinayek, R., D. R. Brown, and R. J. Miller. 1985. Tolerance and cross-tolerance to antisecretory effects of enkephalins on the guineapig ileal mucosa. J. Pharmacol. Exp. Ther. 232:781-785.

8. Fogel, R., and R. B. Kaplan. 1984. Role of enkephalins in regulation of basal intestinal water and ion absorption in the rat. Am. J. Physiol. 246:G386-G392.

9. Kachur, J. F., R. J. Miller, and M. Field. 1980. Control of guinea pig intestinal electrolyte secretion by a delta-opiate receptor. Proc. Natl. Acad. Sci. USA. 77:2753-2756.

10. Hughes, J., T. W. Smith, and N. W. Kosterlitz. 1985. Identification of two related pentapeptides from the brain with potent opiate agonist activity. Nature (Lond.). 258:577-580.

11. Smith, T. W., J. Hughes, H. W. Kosterlitz, and R. P. Sosa. 1986. Enkephalins: isolation, distribution and function. In Opiates and Endogenous Opiate Peptides. H. W. Kosterlitz, editor. Elsevier/ North-Holland Biomedical Press, Amsterdam. 57-62.

12. Furness, J. B., M. Costa, and R. J. Miller. 1983. Distribution and projections of nerves with enkephalin-like immunoreactivity. Neuroscience. 8:653-664.

13. Daniel, E. E., M. Costa, J. B. Furness, and J. R. Kent. 1985. Peptide neurons in the canine small intestine. J. Comp. Neurol. 237:227-238.

14. Polak, J. M., S. R. Bloom, S. N. Sullivan, P. Facer, and A. G. E. Pearse. 1977. Enkephalin-like immunoreactivity in the human gastrointestinal tract. Lancet. i:972-974.

15. Dashwood, M. R., E. S. Degnam, J. Bagnall, and C. S. Thompson. 1985. Autoradiographic localization of opiate receptors in rat small intestine. Eur. J. Pharmacol. 107:267-269.

16. Kachur, J. F., and R. J. Miller. 1982. Characterization of the opiate receptor in the guinea-pig ileal mucosa. Eur. J. Pharmacol. 81:177-183.

17. Nishimura, E., A. M. J. Buchan, and C. H. McIntosh. 1984. Autoradiographic localization of opioid receptors in the rat stomach. Neurosci. Lett. 50:73-78.
18. Jaffe, B. M., A. Ferrara, and D. J. Sherlock. 1986. Comparative effects of ketanserin, atropine, and methysergide on the gastrointestinal effects of hyperserotoninemia in the awake dog. J. Pharmacol. Exp. Ther. 238:536-541.

19. Glass, J., W. C. Chan, and A. R. Gintzler. 1986. Direct analysis of the release of methionine-enkephalin from guinea pig myenteric plexus. Modulation by endogenous opioids and exogenous morphine. J. Pharmacol. Exp. Ther. 239:742-747.

20. Glass, J., D. Clonet, and A. R. Gintzler. 1986. Short term nerve stimulation increases enkephalin production and content in the guinea pig myenteric plexus. Brain Res. 372:180-184.

21. Clark, S. J., and J. W. Smith. 1983. The release of met-enkephalin from the guinea pig ileum at rest and during peristalitic activity. Life Sci. 33:465-468.

22. Clark, S. J., and T. W. Smith. 1981. Peristalsis abolishes the release of methionine-enkephalin from guinea-pig ileum in vitro. Eur. J. Pharmacol. 70:421-424.

23. Clarke, A. M., M. Miller, and R. Shields. 1967. Intestinal transport of sodium, potassium, and water in the dog during sodium depletion. Gastroenterology. 52:846-858.

24. Gleeson, M. H., J. Cullen, and R. H. Dowling. 1972. Intestinal structure and function after small bowel by-pass in the rat. Clin. Sci. 43:731-742.

25. Ferrara, A., M. J. Zinner, and B. M. Jaffe. 1987. Intraluminal release of serotonin, substance $P$, and gastrin in the canine small intestine. Dig. Dis. Sci. 32:289-294.

26. Ruiz-Lopez, M. P., E. Arilla, A. Gomez-Pan, and J. C. Prieto. 1985. Interaction of leu-enkephalin with isolated enterocytes from guinea pig: binding to specific receptors and stimulation of cAMP accumulation. Biochem. Biophys. Res. Commun. 126:404-411.

27. Schiller, L. R., G. R. Davis, C. A. Santa Ana, S. G. Morawski, and J. S. Fordtran. 1982. Studies of the mechanism of the anti-diarrheal effect of codeine. J. Clin. Invest. 70:999-1008.

28. Lee, M. K., and I. M. Coupar. 1980. Opiate receptor mediated inhibition of rat jejunal fluid secretion. Life Sci. 27:2319-2325. 